\title{
A New Method of Numerical Integration of Differential Equations
}

By W. H. Witty

1. Introduction. One of the classical methods of solution to the differential equation

$$
y^{\prime}=f(x, y), \quad y\left(x_{0}\right)=y_{0}
$$

is Heun's method. However, this method requires two substitutions into the differential equation (1) for each step forward. A method described by Lotkin [1] achieves comparable accuracy but requires only one substitution in (1). This can be quite a saving in time when (1) is a complicated function. This article illustrates another method which also requires only one substitution in (1).

2. Heun's method or modified Euler method. With no loss of generality, we will denote the $n-1$ th, $n$ th, $n+\frac{1}{2}$ th, and $n+1$ th values of the variables by the subscripts $-1,0, \frac{1}{2}$, and 1 . Heun's formulas are then:

$$
\begin{aligned}
\bar{y}_{1} & =y_{0}+h y_{0}{ }^{\prime}, \\
\bar{y}_{1}^{\prime} & =f\left(x_{1}, \bar{y}_{1}\right), \\
y_{1} & =y_{0}+\frac{1}{2} h\left(y_{0}{ }^{\prime}+\bar{y}_{1}{ }^{\prime}\right) .
\end{aligned}
$$

If we assume that the values of $y_{0}$ and $y_{0}^{\prime}$ are correct then the error term [1] is

$$
e_{H}=-\frac{1}{24} h^{3}\left[-2 y^{\prime \prime \prime}+6 f_{y} y^{\prime \prime}\right]_{0}+O_{H}\left(h^{4}\right),
$$

where $h$ is the integration interval, $O_{H}\left(h^{4}\right)$ are error terms containing $h^{4}$, and $f_{y}$ is evaluated at $x_{1}$.

3. The method discussed by Lotkin. The formulas of this method are:

$$
\begin{aligned}
y_{1 / 2} & =y_{0}+\frac{1}{2}\left(y_{0}-y_{-1}\right) \\
y_{1 / 2}^{\prime} & =f\left(x_{1 / 2}, y_{1 / 2}\right) \\
y_{1} & =y_{0}+h y_{1 / 2}^{\prime} .
\end{aligned}
$$

Assuming the values $y_{0}$ and $y_{-1}$ to be correct, the error term [1] is

$$
e_{L}=-\frac{1}{24} h^{3}\left[y^{\prime \prime \prime}+9 f_{y} y^{\prime \prime}\right]_{0}+O_{L}\left(h^{4}\right),
$$

where again $h$ is the integration interval, $O_{L}\left(h^{4}\right)$ are error terms containing $h^{4}$, but $f_{y}$ is evaluated at $x_{1 / 2}$.

4. This method. The formulas are:

$$
\begin{aligned}
& y_{1 / 2}=y_{0}+\frac{1}{2} h y_{0}^{\prime}, \\
& y_{1 / 2}^{\prime}=f\left(x_{1 / 2}, y_{1 / 2}\right),
\end{aligned}
$$

Received October 15, 1963. 


$$
\begin{aligned}
& y_{1}=y_{0}+h y_{1 / 2}^{\prime}, \\
& y_{1}^{\prime}=2 y_{1 / 2}^{\prime}-y_{0}^{\prime} .
\end{aligned}
$$

These formulas show in this method extrapolation is used to determine $y_{1}^{\prime}$, whereas in the second method extrapolation is used to determine $y_{1 / 2}$. Also it is not required to know the numerical value of $y_{-1}$.

To find the error in this method we first assume $z(x)$ to be the true solution of (1). Therefore

$$
z_{1 / 2}=z_{0}+\frac{1}{2} h z_{0}^{\prime}+\frac{1}{8} h^{2} z_{0}^{\prime \prime}+\frac{1}{48} h^{3} z_{0}^{\prime \prime \prime}+\cdots .
$$

Since $y_{0}=z_{0}, y_{0}^{\prime}=z_{0}^{\prime}$ if we subtract (14) from (10) and retain only the $h^{2}$ and $h^{3}$ terms, we get the following error:

$$
e_{1}=y_{1 / 2}-z_{1 / 2}=-\frac{1}{8} h^{2} z_{0}^{\prime \prime}-\frac{1}{48} h^{3} z_{0}^{\prime \prime \prime} .
$$

Also, since

$$
z_{1 / 2}^{\prime}=f\left(x_{1 / 2}, z_{1 / 2}\right),
$$

we get, by subtracting (16) from (11),

$$
e_{2}=y_{1 / 2}^{\prime}-z_{1 / 2}^{\prime}=f\left(x_{1 / 2}, y_{1 / 2}\right)-f\left(x_{1 / 2}, z_{1 / 2}\right),
$$

which by the mean value theorem is

$$
\begin{aligned}
& e_{2}=y_{1 / 2}^{\prime}-z_{1 / 2}^{\prime}=f_{y}\left(x_{1 / 2}, y\right)\left(y_{1 / 2}-z_{1 / 2}\right), \\
& e_{2}=y_{1 / 2}^{\prime}-z_{1 / 2}^{\prime}=f_{y} e_{1}
\end{aligned}
$$

where $f_{y}=f_{y}\left(x_{1 / 2}, y\right)$. From (12) and (17) we have

$$
\begin{aligned}
& y_{1}=z_{0}+h\left[z_{1 / 2}^{\prime}+f_{y} e_{1}\right] \\
& y_{1}=z_{0}+h\left[z_{0}^{\prime}+\frac{1}{2} h z_{0}^{\prime \prime}+\frac{1}{8} h^{2} z_{0}^{\prime \prime \prime}+\frac{1}{48} h^{3} z_{0}^{i v}+\cdots\right]+h f_{y} e_{1}
\end{aligned}
$$

and, since

$$
z_{1}=z_{0}+h z_{0}^{\prime}+\frac{1}{2} h^{2} z_{0}^{\prime \prime}+\frac{1}{6} h^{3} z_{0}^{\prime \prime \prime}+\frac{1}{24} h^{4} z_{0}{ }^{i v}+\cdots,
$$

we subtract (19) from (18) neglecting higher order terms to get

$$
\begin{aligned}
e_{W}= & y_{1}-z_{1}=-\frac{1}{24} h^{3} z_{0}{ }^{\prime \prime \prime}-\frac{1}{48} h^{4} z_{0}^{i v}+h f_{y} e_{1} \\
& =-\frac{1}{24} h^{3} z_{0}{ }^{\prime \prime \prime}-\frac{1}{48} h^{4} z_{0}^{i v}-\frac{1}{8} h^{3} f_{y} z_{0}^{\prime \prime}-\frac{1}{48} h^{4} f_{y} z_{0}{ }^{\prime \prime \prime}, \\
e_{W}= & -\frac{1}{24} h^{3}\left[z^{\prime \prime \prime}+3 f_{y} z^{\prime \prime}\right]_{0}+O_{W}\left(h^{4}\right),
\end{aligned}
$$

where again $h$ is the integration interval, $O_{W}\left(h^{4}\right)$ are error terms containing $h^{4}$, and $f_{y}$ is evaluated at $x_{1 / 2}$. Now in order to make only one substitution in the differential equation, we extrapolate for the $y_{1}^{\prime}$ by equation (13). Substituting (17) in (13) we have

$$
\begin{aligned}
y_{1}^{\prime}= & 2\left[z_{1 / 2}^{\prime}+f_{y} e_{1}\right]-z_{0}^{\prime} \\
& =2\left[z_{0}^{\prime}+\frac{1}{2} h z_{0}^{\prime \prime}+\frac{1}{8} h^{2} z_{0}^{\prime \prime \prime}+\frac{1}{48} h^{3} z_{0}^{i v}+\cdots\right]+2 f_{y} e_{1}-z_{0}^{\prime}, \\
y_{1}^{\prime}= & z_{0}^{\prime}+h z_{0}^{\prime \prime}+\frac{1}{4} h^{2} z_{0}^{\prime \prime \prime}+\frac{1}{24} h^{3} z_{0}^{i v}+\cdots+2 f_{y} e_{1} .
\end{aligned}
$$

Since 


$$
z_{1}^{\prime}=z_{0}^{\prime}+h z_{0}^{\prime \prime}+\frac{1}{2} h^{2} z_{0}^{\prime \prime \prime}+\frac{1}{6} h^{3} z_{0}^{i v}+\cdots,
$$

subtracting (22) from (21), substituting from (15), and neglecting higher order terms, we obtain

$$
e_{3}=y_{1}^{\prime}-z_{1}^{\prime}=-\frac{1}{4} h^{2}\left[z_{0}^{\prime \prime \prime}+f_{y} z_{0}^{\prime \prime}\right]-\frac{1}{24} h^{3}\left[3 z_{0}{ }^{i v}+f_{y} z_{0}^{\prime \prime \prime}\right]
$$

Restating the error terms of the three methods using more generalized subscripts we have

$$
\begin{aligned}
e_{H} & =-\frac{1}{24} h^{3}\left[-2 y^{\prime \prime \prime}+6 f_{y} y^{\prime \prime}\right]_{n}+O_{H}\left(h^{4}\right), \\
e_{L} & =-\frac{1}{24} h^{3}\left[y^{\prime \prime \prime}+9 f_{y} y^{\prime \prime}\right]_{n}+O_{L}\left(h^{4}\right) \\
e_{W} & =-\frac{1}{24} h^{3}\left[y^{\prime \prime \prime}+3 f_{y} y^{\prime \prime}\right]_{n}+O_{W}\left(h^{4}\right)
\end{aligned}
$$

A comparison of (24), (25) and (26) reveals that there are many cases where the third method gives the least error. Also since the last two methods require only one evaluation of (1), we could use an integration integral of one-half the interval used by the first method and greatly increase our accuracy.

5. Comparison of results. Table 1 gives the values obtained from the integration of the differential equation

$$
\mathrm{y}^{\prime}=\left(1+y^{2}\right)^{-1}, \quad y(0)=0
$$

by the three different methods using the same integration interval $h=0.1$. The exact solution of (27) $y^{3}+3 y-3 x=0$ is given in the second column. Values obtained by Heun's method, Lotkin's method and this method are given in the third, fourth, and fifth columns. The resulting errors are given in the sixth, seventh, and eighth columns. The values of the independent variable are given in the first column. In order to compare the three methods so that all compute the differential equation (27) the same number of times, we must use in the last two methods an $h$ which is one-half that used in Heun's method. Results of using $h=.05$ in the last two methods are shown in Table 2.

TABLE 1

Comparison of Results

\begin{tabular}{c|c|c|c|c|c|c|c}
\hline$x$ & $\begin{array}{c}\text { Exact } \\
\text { solution }\end{array}$ & Heun & Lotkin & $\begin{array}{c}\text { This } \\
\text { method }\end{array}$ & $E_{H}$ & $E_{L}$ & $E_{W}$ \\
\cline { 1 - 5 } & .00000 & .00000 & .00000 & .00000 & $0 \cdot 10^{-5}$ & $0 \cdot 10^{-5}$ & $0 \cdot 10^{-5}$ \\
& .09967 & .09950 & .09975 & .09975 & -17 & 8 & 8 \\
.2 & .19744 & .19712 & .19756 & .19756 & -32 & 12 & 12 \\
.3 & .29172 & .29129 & .29184 & .29187 & -43 & 12 & 15 \\
.4 & .38149 & .38097 & .38153 & .38161 & -52 & 4 & 12 \\
.5 & .46622 & .46564 & .46615 & .46631 & -58 & -7 & 9 \\
.6 & .54580 & .54519 & .54560 & .54583 & -61 & -20 & 3 \\
.7 & .62040 & .61977 & .62009 & .62039 & -63 & -31 & -1 \\
.8 & .69033 & .68971 & .68991 & .69026 & -62 & -42 & -7 \\
.9 & .75597 & .75536 & .75547 & .75588 & -61 & -50 & -9 \\
1.0 & .81773 & .81712 & .81715 & .81758 & -61 & -58 & -15 \\
\hline
\end{tabular}


TABLE 2

Comparison of Results

\begin{tabular}{c|c|c|c|c}
\hline$x$ & Lotkin & This method & $E_{L}$ & $E_{W}$ \\
\cline { 2 - 3 } 0 & .00000 & .00000 & $0 \cdot 10^{-5}$ & $0 \cdot 10^{-5}$ \\
.1 & .09969 & .09969 & 2 & 2 \\
.2 & .19746 & .19747 & 2 & 3 \\
.3 & .29175 & .29176 & 3 & 4 \\
.4 & .38150 & .38152 & 1 & 3 \\
.5 & .46620 & .46624 & -2 & 2 \\
.6 & .54575 & .54581 & -5 & 1 \\
.7 & .62032 & .62040 & -8 & 0 \\
.8 & .69023 & .69032 & -10 & -1 \\
.9 & .75585 & .75595 & -12 & -2 \\
1.0 & .81759 & .81769 & -14 & -4 \\
\hline
\end{tabular}

6. Conclusions. Since this method and Lotkin's method require only one evaluation of the differential equation for each step forward as compared to two evaluations by Heun's method, it is evident that a great deal of time can be saved by the use of these methods instead of Heun's method. The practicality of this, however, would depend upon whether the accuracy of these methods was comparable to that of Heun's method.

If we compare these two methods with Heun's method on the basis of the same number of evaluations of the differential equation, we may use an integration interval one-half that used by Heun's method. In effect the error terms (25) and (26) become $1 / 8$ of their former values. This gives greater accuracy, but the time required to solve the differential equations would be the same for all three methods.

Comparing this method with Lotkin's method, we see that the primary difference is that extrapolation is used to obtain the derivative rather than the variable. The extrapolation formula (13) is obtained from Newton's interpolation formula. Euler's formula is used to obtain the values of the variable at the half step in this method. This seems to be a more natural method than extrapolation using backword values because initial conditions for the independent and dependent variables are usually known, and the initial value of the derivative can be calculated from the differential equation. The backward value required by Lotkin's method is sometimes not known.

It is apparent from analysis of (24), (25), (26) and the tables that there exist differential equations which can be integrated numerically more accurately by this method than by either Heun's or Lotkin's method.

Marshall Space Flight Center

Redstone Arsenal, Alabama

1. M. Lotkin, "A note on the midpoint method of integration," J. Assoc. Comput. Mach., v. 3,1956, p. $208-211$. 